\title{
On Entire and Meromorphic Functions That Share One Small Function with Their Differential Polynomial
}

\author{
Subhas S. Bhoosnurmath and Smita R. Kabbur \\ Department of Mathematics, Karnatak University, Dharwad 580003, India \\ Correspondence should be addressed to Subhas S. Bhoosnurmath; ssbmath@gmail.com
}

Received 24 September 2012; Accepted 22 December 2012

Academic Editor: Mats Ehrnström

Copyright (c) 2013 S. S. Bhoosnurmath and S. R. Kabbur. This is an open access article distributed under the Creative Commons Attribution License, which permits unrestricted use, distribution, and reproduction in any medium, provided the original work is properly cited.

We study the uniqueness of meromorphic functions that share one small function with more general differential polynomial $P[f]$. As corollaries, we obtain results which answer open questions posed by $\mathrm{Yu}$ (2003).

\section{Introduction and Main Results}

In this paper, a meromorphic functions mean meromorphic in the whole complex plane. We use the standard notations of Nevanlinna theory (see [1]). A meromorphic function $a(z)$ is called a small function with respect to $f(z)$ if $T(r, a)=$ $S(r, f)$, that is, $T(r, a)=o(T(r, f))$ as $r \rightarrow \infty$ possibly outside a set of finite linear measure. If $f(z)-a(z)$ and $g(z)-a(z)$ have the same zeros with same multiplicities (ignoring multiplicities), then we say that $f(z)$ and $g(z)$ share $a(z)$ CM (IM).

For any constant $a$, we denote by $N_{k)}(r, 1 /(f-a))$ the counting function for zeros of $f(z)-a$ with multiplicity no more than $k$ and $\bar{N}_{k)}(r, 1 /(f-a))$ the corresponding for which multiplicity is not counted. Let $N_{(k}(r, 1 /(f-a))$ be the counting function for zeros of $f(z)-a$ with multiplicity at least $k$ and $\bar{N}_{(k}(r, 1 /(f-a))$ the corresponding for which the multiplicity is not counted.

Let $f$ and $g$ be two nonconstant meromorphic functions sharing value 1IM. Let $z_{0}$ be common one point of $f$ and $g$ with multiplicity $p$ and $q$, respectively. We denote by $N_{L}(r, 1 /(f-1))\left(\bar{N}_{L}(r, 1 /(f-1))\right)$ the counting (reduced) function of those 1 points of $f$ where $p>q$; by $N_{E}^{1)}(r, 1 /(f-$ 1)) the counting function of those 1-points of $f$ where $p=$ $q=1$; by $N_{E}^{(2}(r, 1 /(f-1))$ the counting function of those $1-$ points of $f$ where $p=q \geq 2$. In the same way, we can define
$N_{L}(r, 1 /(g-1)), N_{E}^{1)}(r, 1 /(g-1))$ and $N_{E}^{(2}(r, 1 /(g-1))$ (see $[2])$.

In 1996, Brück [3] posed the following conjecture.

Conjecture 1. Let $f$ be a nonconstant entire function such that the hyper-order $\sigma_{2}(f)$ of $f$ is not a positive integer and $\sigma_{2}(f)<$ $\infty$. If $f$ and $f^{\prime}$ share a finite value a CM, then $\left(f^{\prime}-a\right) /(f-$ $a)=c$, where $c$ is a nonzero constant.

In [3], under an additional hypothesis, Brück proved that the conjecture holds when $a=1$.

Theorem A. Let $f$ be a nonconstant entire function. If $f$ and $f^{\prime}$ share the value $1 C M$ and if $N\left(r, 1 / f^{\prime}\right)=S(r, f)$, then $\left(f^{\prime}-\right.$ $1) /(f-1)=c$, for some constant $c \in \mathbb{C} \backslash\{0\}$.

Many people extended this theorem and obtained many results. In 2003, Yu [4] proved the following theorem.

Theorem B. Let $k \geq 1$. Let $f$ be a nonconstant meromorphic function and $a(z)$ a meromorphic function such that $a(z) \not \equiv$ $0, \infty, f$ and $a$ do not have any common pole and $T(r, a)=$ $o(T(r, f))$ as $r \rightarrow \infty$. If $f-a$ and $f^{(k)}-a$ share the value $0 C M$ and

$$
4 \delta(0, f)+2(8+k) \Theta(\infty, f)>19+2 k,
$$

then $f \equiv f^{(k)}$. 
Theorem C. Let $k \geq 1$. Let $f$ be a nonconstant entire function and $a(z)$ be a meromorphic function such that $a(z) \neq \equiv, \infty$ and $T(r, a)=o(T(r, f))$ as $r \rightarrow \infty$. If $f-a$ and $f^{(k)}-a$ share the value 0 CM and

$$
\delta(0, f)>\frac{3}{4}
$$

then $f \equiv f^{(k)}$. tions.

In the same paper, the author posed the following ques-

Question 1. Can a CM shared value be replaced by an IM shared value in Theorem C?

Question 2. Is the condition $\delta(0, f)>3 / 4$ sharp in Theorem $\mathrm{C}$ ?

Question 3. Is the condition $4 \delta(0, f)+2(8+k) \Theta(\infty, f)>$ $19+2 k$ sharp in Theorem B?

In 2004, Liu and $\mathrm{Gu}$ [5] applied different method and obtained the following theorem which answers some questions posed in [4].

Theorem D. Let $k \geq 1$. Let $f$ be a nonconstant meromorphic function and $a(z)$ a meromorphic function such that $a(z) \quad \equiv$ $0, \infty$ and $T(r, a)=S(r, f)$ as $r \rightarrow \infty$. If $f-a$ and $f^{(k)}-a$ share the value $0 C M$ and $f^{(k)}$ and $a(z)$ do not have any common poles of same multiplicity and

$$
2 \delta(0, f)+4 \Theta(\infty, f)>5
$$

then $f \equiv f^{(k)}$.

Theorem E. Let $k \geq 1$. Let $f$ be a nonconstant entire function and $a(z)$ a meromorphic function such that $a(z) \quad \neq \equiv, \infty$ and $T(r, a)=S(r, f)$ as $r \rightarrow \infty$. If $f-a$ and $f^{(k)}-a$ share the value $0 \mathrm{CM}$ and

$$
\delta(0, f)>\frac{1}{2}
$$

then $f \equiv f^{(k)}$.

Recently, Zhang and Lü [6] considered the problem of meromorphic functions sharing one small function with its $k$ th derivative and proved the following theorem.

Theorem F. Let $k(\geq 1), n(\geq 1)$ be integers and $f$ a nonconstant meromorphic function. Also let $a(z) \neq \equiv, \infty$ be a small meromorphic function with respect to $f$. If $f^{n}$ and $f^{(k)}$ share the value $a(z)$ IM and

$$
(2 k+6) \Theta(\infty, f)+4 \Theta(0, f)+2 \delta_{k+2}(0, f)>2 k+12-n
$$

or $f^{n}$ and $f^{(k)}$ share the value $a(z) C M$ and

$$
(k+3) \Theta(\infty, f)+2 \Theta(0, f)+\delta_{k+2}(0, f)>k+6-n,
$$

then $f \equiv f^{(k)}$.
Regarding these results, a natural question is what can be said when a nonconstant meromorphic function $f$ shares one nonzero small meromorphic function $a(z)$ with $P[f]$, where $P[f]$ is a differential polynomial in $f$.

Definition 2. Any expression of the type

$$
P[f]=\sum_{i=1}^{n} \alpha_{i}(z) f^{n_{i_{0}}}\left(f^{\prime}\right)^{n_{i_{1}}}\left(f^{\prime \prime}\right)^{n_{i_{2}}} \cdots\left(f^{(m)}\right)^{n_{i_{m}}}
$$

is called differential polynomial in $f$ of degree $\bar{d}(P)$, lower degree $\underline{d}(P)$, and weight $\Gamma_{P}$, where $n_{i_{0}}, n_{i_{1}}, \ldots, n_{i_{m}}$ are nonnegative integers, $\alpha_{i}=\alpha_{i}(z)$ are meromorphic functions satisfying $T\left(r, \alpha_{i}\right)=S(r, f)$ and

$$
\begin{gathered}
\bar{d}(P)=\max \left\{\sum_{j=0}^{m} n_{i j}: 1 \leq i \leq n\right\}, \\
\underline{d}(P)=\min \left\{\sum_{j=0}^{m} n_{i j}: 1 \leq i \leq n\right\}, \\
\Gamma_{P}=\max \left\{\sum_{j=0}^{m}(j+1) n_{i j}: 1 \leq i \leq n\right\} .
\end{gathered}
$$

Further, if $\bar{d}(P)=\underline{d}(P)=n$ (say), then the differential polynomial $P[f]$ is called a homogeneous differential polynomial in $f$ of degree $n$.

Correspond to the above question, we obtain the following results, which extend and improve Theorems A-F and give answers to the questions possed by $\mathrm{Yu}$ [4] for more general differential polynomial.

Theorem 3. Let $f$ be a nonconstant meromorphic function and $a(z)$ be a small meromorphic function such that $a(z)$ \# $0, \infty$. $P[f]$ be a nonconstant differential polynomial in $f$ as defined in (7). If $f$ and $P[f]$ share the value $a I M$ and

$$
\begin{gathered}
(2 Q+6) \Theta(\infty, f)+(2+3 \underline{d}(P)) \delta(0, f) \\
>2 Q+2 \underline{d}(P)+\bar{d}(P)+7
\end{gathered}
$$

then $f \equiv P[f]$.

Remark 4. Taking $P[f]=f^{(k)}$, that is, $Q=k, \bar{d}(P)=$ $\underline{d}(P)=1$ in $(9)$, we get $(2 k+6) \Theta(\infty, f)+5 \delta(0, f)>2 k+10$, which improves (5) and extends the theorem to more general differential polynomial $P[f]$ as defined in (7).

Theorem 5. Let $f$ be a nonconstant meromorphic function and $a(z)$ be a small meromorphic function such that $a(z)$ \# $0, \infty$. Let $P[f]$ a nonconstant differential polynomial in $f$ as defined in (7). If $f$ and $P[f]$ share the value $a C M$ and

$$
3 \Theta(\infty, f)+(\underline{d}(P)+1) \delta(0, f)>4,
$$

then $f \equiv P[f]$. 
Remark 6. Taking $P[f]=f^{(k)}$, that is, $Q=k, \bar{d}(P)=\underline{d}(P)=$ 1 in $(10)$, we get $3 \Theta(\infty, f)+2 \delta(0, f)>4$, which improves (6) and extends the theorem to more general differential polynomial $P[f]$ as defined in (7).

Remark 6 gives answer to Question 3 of [4].

Theorem 7. Let $f$ be a nonconstant entire function and $a(z) a$ small meromorphic function such that $a(z) \not \equiv 0, \infty$. Let $P[f]$ be a nonconstant differential polynomial in $f$ as defined in (7) If $f$ and $P[f]$ share the value a IM and

$$
(3 \underline{d}(P)+2) \delta(0, f)>2 \bar{d}(P)+2,
$$

then $f \equiv P[f]$.

Remark 8. Taking $P[f]=f^{(k)}$, that is, $Q=k, \bar{d}(P)=\underline{d}(P)=$ 1 in (11), we get

$$
\delta(0, f)>\frac{4}{5}
$$

Remark 8 gives answer to Question 1 of $\mathrm{Yu}$ [4].

Theorem 9. Let $f$ be a nonconstant entire function and $a(z)$ be a small meromorphic function such that $a(z) \not \equiv 0, \infty . P[f]$ be a nonconstant differential polynomial in $f$ as defined in (7). If $f$ and $P[f]$ share the value $a C M$ and

$$
(\underline{d}(P)+1) \delta(0, f)>1,
$$

then $f \equiv P[f]$.

Remark 10. Taking $P[f]=f^{(k)}$, that is, $Q=k, \bar{d}(P)=\underline{d}(P)=$ 1 in (13), we get $\delta(0, f)>1 / 2$, which improves Theorem $\mathrm{C}$ and extends the theorem to more general differential polynomial $P[f]$ as defined in (7).

Remark 10 gives answer to Question 2 of Yu [4].

Remark 11. By proving Remarks 6, 8, and 10 we have answered Questions 3, 1, and 2 (of [4]), respectively, for the case $f^{(k)}$. Theorems 3-9 improve and generalize Theorems A-F for more general differential polynomial $P[f]$.

\section{Lemma}

Lemma 12 (see [7]). Let $f$ ba a meromorphic function and $P[f]$ be a differential polynomial in $f$. Then

$$
\begin{gathered}
m\left(r, \frac{P[f]}{f^{\bar{d}(P)}}\right) \\
\leq(\bar{d}(P)-\underline{d}(P)) m\left(r, \frac{1}{f}\right)+S(r, f), \\
m\left(r, \frac{P[f]}{f \underline{d}(P)}\right) \\
\leq(\bar{d}(P)-\underline{d}(P)) m(r, f)+S(r, f), \\
N\left(r, \frac{P[f]}{f^{\bar{d}}(P)}\right) \leq(\bar{d}(P)-\underline{d}(P)) N\left(r, \frac{1}{f}\right) \\
N(r, P[f] \leq \bar{d}(P) N(r, f)+Q \bar{N}(r, f)+S(r, f), \\
T(r, P(f)) \leq Q \bar{N}(r, f)+\bar{d}(P) T(r, f)+S(r, f),
\end{gathered}
$$

where $Q=\max \left\{n_{i_{1}}+2 n_{i_{2}}+3 n_{i_{3}}+\cdots+m n_{i_{m}} ; 1 \leq i \leq n\right\}$.

Lemma 13 (see [8]). Let $f$ be a nonconstant meromorphic function, then

$$
\begin{gathered}
N\left(r, \frac{1}{f^{(k)}}\right) \leq T\left(r, f^{(k)}\right)-T(r, f)+N\left(r, \frac{1}{f}\right)+S(r, f) \\
N\left(r, \frac{1}{f^{(k)}}\right) \leq N\left(r, \frac{1}{f}\right)+k \bar{N}(r, f)+S(r, f) .
\end{gathered}
$$

Lemma 14 (see [9]). Let

$$
H=\left(\frac{F^{\prime \prime}}{F^{\prime}}-\frac{2 F^{\prime}}{F-1}\right)-\left(\frac{G^{\prime \prime}}{G^{\prime}}-\frac{2 G^{\prime}}{G-1}\right),
$$

where $F$ and $G$ are two nonconstant meromorphic functions. If $F$ and $G$ share 1 IM and $H \neq \equiv 0$, then

$$
N_{E}^{1)}\left(r, \frac{1}{F-1}\right) \leq N(r, H)+S(r, F)+S(r, G) .
$$


Lemma 15. Let $f$ be a transcendental meromorphic function. Let $P[f]$ be defined as in (7). If $P[f] \neq 0$, we have

$$
\begin{gathered}
N\left(r, \frac{1}{P[f]}\right) \\
\leq T(r, P[f])-T\left(r, f^{\bar{d}(P)}\right) \\
+(\bar{d}(P)-\underline{d}(P)) m\left(r, \frac{1}{f}\right) \\
+N\left(r, \frac{1}{f \bar{d}(P)}\right)+S(r, f), \\
N\left(r, \frac{1}{P[f]}\right) \leq Q \bar{N}(r, f)+(\bar{d}(P)-\underline{d}(P)) m\left(r, \frac{1}{f}\right) \\
+N\left(r, \frac{1}{f^{\bar{d}(P)}}\right)+S(r, f) .
\end{gathered}
$$

Proof. By the first fundamental theorem, we have

$$
N\left(r, \frac{1}{P[f]}\right)=T(r, P[f])-m\left(r, \frac{1}{P[f]}\right)+O(1) .
$$

We have

$$
\begin{gathered}
m\left(r, \frac{1}{f^{\bar{d}(P)}}\right) \leq m\left(r, \frac{P[f]}{f^{\bar{d}(P)}}\right)+m\left(r, \frac{1}{P[f]}\right) \\
m\left(r, \frac{1}{f^{\bar{d}(P)}}\right)-m\left(r, \frac{P[f]}{f^{\bar{d}(P)}}\right) \leq m\left(r, \frac{1}{P[f]}\right) \\
-m\left(r, \frac{1}{f^{\bar{d}(P)}}\right)+m\left(r, \frac{P[f]}{f^{\bar{d}(P)}}\right) \geq-m\left(r, \frac{1}{P[f]}\right)
\end{gathered}
$$

or

$$
-m\left(r, \frac{1}{P[f]}\right) \leq-m\left(r, \frac{1}{f^{\bar{d}(P)}}\right)+m\left(r, \frac{P[f]}{f^{\bar{d}}(P)}\right) .
$$

By (21), (23) and Lemma 12, we obtain (19).

Since

$$
\begin{aligned}
& T(r, P[f]) \\
&= m(r, P[f])+N(r, P[f]) \\
& \leq m\left(r, \frac{P[f]}{f^{\underline{d}(P)}}\right)+m\left(r, f^{\underline{d}(P)}\right)+N(r, P[f]) \\
& \leq(\bar{d}(P)-\underline{d}(P)) m(r, f)+\underline{d}(P) m(r, f) \\
&+\bar{d}(P) N(r, f)+Q \bar{N}(r, f)+S(r, f) \\
& \leq \bar{d}(P) m(r, f)+\bar{d}(P) N(r, f) \\
&+Q \bar{N}(r, f)+S(r, f),
\end{aligned}
$$

we get

$$
T(r, P[f]) \leq \bar{d}(P) T(r, f)+Q \bar{N}(r, f)+S(r, f) .
$$

Substituting (25) in (19), we obtain (20).

Lemma 16 (see [10]). Let $f$ be a transcendental meromorphic function, $P[f]$ a differential polynomial in $f$ of degree $\bar{d}(P)$ and weight $\Gamma_{P}$. Then $T(r, P)=O(T(r, f)), S(r, P)=S(r, f)$.

\section{Proof of Theorems}

Proof of Theorem 3. Let

$$
F=\frac{P[f]}{a}, \quad G=\frac{f}{a} .
$$

From the conditions of Theorem 3, we know that $F$ and $G$ share 1 IM. From (26), we have

$$
\begin{gathered}
T(r, F)=O(T(r, f))+S(r, f), T(r, G) \\
\leq T(r, f)+S(r, f), \\
\bar{N}(r, F)=\bar{N}(r, G)+S(r, f), \\
\bar{N}(r, F)=\bar{N}(r, f)+S(r, f), \bar{N}(r, G) \\
=\bar{N}(r, f)+S(r, f), \\
N_{E}^{1)}\left(r, \frac{1}{F-1}\right)=N_{E}^{1)}\left(r, \frac{1}{G-1}\right)+S(r, f), \\
\bar{N}_{E}^{(2}\left(r, \frac{1}{F-1}\right)=\bar{N}_{E}^{(2}\left(r, \frac{1}{G-1}\right)+S(r, f), \\
\bar{N}_{L}\left(r, \frac{1}{F-1}\right) \leq \bar{N}\left(r, \frac{1}{F}\right)+\bar{N}(r, F)+S(r, f), \\
\bar{N}_{(}\left(r, \frac{1}{F-1}\right) \\
=\bar{N}\left(r, \frac{1}{G-1}\right)+S(r, f) \\
\leq N_{E}^{1)}\left(r, \frac{1}{F-1}\right)+\bar{N}_{E}^{(2}\left(r, \frac{1}{F-1}\right) \\
+N_{L}\left(r, \frac{1}{F-1}\right)+N_{L}\left(r, \frac{1}{G-1}\right)+S(r, f) .
\end{gathered}
$$

Let $H$ be defined by (17). Suppose that $H \neq \equiv \quad 0$. By Lemma 14, (18) holds.

From (17) and (28), we have

$$
\begin{aligned}
N(r, H) \leq & N_{(2}\left(r, \frac{1}{F}\right)+N_{(2}\left(r, \frac{1}{G}\right)+\bar{N}(r, G) \\
& +N_{L}\left(r, \frac{1}{F-1}\right)+N_{L}\left(r, \frac{1}{G-1}\right) \\
& +N_{0}\left(r, \frac{1}{F^{\prime}}\right)+N_{0}\left(r, \frac{1}{G^{\prime}}\right),
\end{aligned}
$$

where $N_{0}\left(r, 1 / F^{\prime}\right)$ denotes the counting function corresponding to the zeros of $F^{\prime}$ which are not the zeros of $F$ and $F-1$. Similarly, $N_{0}\left(r, 1 / G^{\prime}\right)$ is defined. 
From the second fundamental theorem, we have

$$
\begin{aligned}
T(r, F) & +T(r, G) \\
\leq & \bar{N}(r, F)+\bar{N}\left(r, \frac{1}{F}\right)+\bar{N}(r, G) \\
& +\bar{N}\left(r, \frac{1}{G}\right)+\bar{N}\left(r, \frac{1}{F-1}\right)+\bar{N}\left(r, \frac{1}{G-1}\right) \\
& -N_{0}\left(r, \frac{1}{F^{\prime}}\right)-N_{0}\left(r, \frac{1}{G^{\prime}}\right)+S(r, f) .
\end{aligned}
$$

Since $F$ and $G$ share $1 \mathrm{IM}$, we get from (33):

$$
\begin{aligned}
\bar{N}\left(r, \frac{1}{F-1}\right)+\bar{N}\left(r, \frac{1}{G-1}\right) \\
=2 N_{E}^{1)}\left(r, \frac{1}{F-1}\right)+2 N_{L}\left(r, \frac{1}{F-1}\right) \\
\quad+2 N_{L}\left(r, \frac{1}{G-1}\right)+2 \bar{N}_{E}^{(2}\left(r, \frac{1}{F-1}\right) .
\end{aligned}
$$

From this, (18), and (34), we have

$$
\begin{aligned}
& \bar{N}\left(r, \frac{1}{F-1}\right)+\bar{N}\left(r, \frac{1}{G-1}\right) \\
& \quad \leq N_{(2}\left(r, \frac{1}{F}\right)+N_{(2}\left(r, \frac{1}{G}\right)+\bar{N}(r, G)+N_{0}\left(r, \frac{1}{F^{\prime}}\right) \\
& \quad+3 N_{L}\left(r, \frac{1}{G-1}\right)+3 N_{L}\left(r, \frac{1}{F-1}\right)+N_{E}^{1)}\left(r, \frac{1}{F-1}\right) \\
& \quad+2 \bar{N}_{E}^{(2}\left(r, \frac{1}{G-1}\right)+N_{0}\left(r, \frac{1}{G^{\prime}}\right)+S(r, f) .
\end{aligned}
$$

It is clear that

$$
\begin{aligned}
N_{L} & \left(r, \frac{1}{F-1}\right)+2 N_{L}\left(r, \frac{1}{G-1}\right) \\
& +2 \bar{N}_{E}^{(2}\left(r, \frac{1}{G-1}\right)+N_{E}^{1)}\left(r, \frac{1}{F-1}\right) \\
\leq & N\left(r, \frac{1}{G-1}\right) \\
\leq & T(r, G)+O(1) .
\end{aligned}
$$

Combining (37), and (38), we obtain

$$
\begin{aligned}
& \bar{N}\left(r, \frac{1}{F-1}\right)+\bar{N}\left(r, \frac{1}{G-1}\right) \\
& \leq N_{(2}\left(r, \frac{1}{F}\right)+N_{(2}\left(r, \frac{1}{G}\right)+\bar{N}(r, G) \\
&+2 N_{L}\left(r, \frac{1}{F-1}\right)+N_{L}\left(r, \frac{1}{G-1}\right)+T(r, G) \\
&+N_{0}\left(r, \frac{1}{F^{\prime}}\right)+N_{0}\left(r, \frac{1}{G^{\prime}}\right)+S(r, f) .
\end{aligned}
$$

Substituting (39) in (35) and using (28), we obtain

$$
\begin{aligned}
T(r, F) \leq & 3 \bar{N}(r, G)+N\left(r, \frac{1}{F}\right)+N\left(r, \frac{1}{G}\right) \\
& +2 N_{L}\left(r, \frac{1}{F-1}\right)+N_{L}\left(r, \frac{1}{G-1}\right)+S(r, f) .
\end{aligned}
$$

Using (26) and (19), we get

$$
\begin{aligned}
\bar{d}(P) & T(r, f) \\
\leq & 3 \bar{N}(r, G)+(\bar{d}(P)-\underline{d}(P)) m\left(r, \frac{1}{f}\right) \\
& +N\left(r, \frac{1}{f}\right)+N\left(r, \frac{1}{f \bar{d}(P)}\right) \\
& +2 N_{L}\left(r, \frac{1}{F-1}\right)+N_{L}\left(r, \frac{1}{G-1}\right)+S(r, f) .
\end{aligned}
$$

From (16), (20), and (26) we have

$$
\begin{aligned}
2 N_{L} & \left(r, \frac{1}{F-1}\right)+N_{L}\left(r, \frac{1}{G-1}\right) \\
\leq & 2 N\left(r, \frac{1}{F^{\prime}}\right)+N\left(r, \frac{1}{G^{\prime}}\right) \\
\leq & 2\left[N\left(r, \frac{1}{F}\right)+\bar{N}(r, F)\right]+N\left(r, \frac{1}{f}\right) \\
& +\bar{N}(r, f)+S(r, f) \\
\leq & 2 N\left(r, \frac{1}{P[f]}\right)+3 \bar{N}(r, f) \\
& +N\left(r, \frac{1}{f}\right)+S(r, f) \\
\leq & 2 Q \bar{N}(r, f)+2 N\left(r, \frac{1}{f^{\bar{d}}(P)}\right) \\
& +2(\bar{d}(P)-\underline{d}(P)) m\left(r, \frac{1}{f}\right) \\
& +3 \bar{N}(r, f)+N\left(r, \frac{1}{f}\right)+S(r, f) \\
\leq & (2 Q+3) \bar{N}(r, f)+(2 \bar{d}(P)+1) N\left(r, \frac{1}{f}\right) \\
& +2(\bar{d}(P)-\underline{d}(P)) m\left(r, \frac{1}{f}\right)+S(r, f) .
\end{aligned}
$$


From (41) and (42), we get

$$
\begin{aligned}
\bar{d}(P) T(r, f) \\
\leq(2 Q+6) \bar{N}(r, f)+(2+3 \bar{d}(P)) N\left(r, \frac{1}{f}\right) \\
\quad+3(\bar{d}(P)-\underline{d}(P)) m\left(r, \frac{1}{f}\right)+S(r, f) \\
\leq(2 Q+6) \bar{N}(r, f)+(2+3 \underline{d}(P)) N\left(r, \frac{1}{f}\right) \\
\quad+3(\bar{d}(P)-\underline{d}(P)) T\left(r, \frac{1}{f}\right)+S(r, f) \\
(3 \underline{d}(P)-2 \bar{d}(P)) T(r, f) \\
\leq \quad(2 Q+6) \bar{N}(r, f)+(2+3 \underline{d}(P)) N\left(r, \frac{1}{f}\right)+S(r, f) \\
\leq\{(2 Q+6)(1-\Theta(\infty, f))+(2+3 \underline{d}(P))(1-\delta(0, f))\} \\
\quad \times T(r, f)+S(r, f) \\
\leq\{(2 Q+3 \underline{d}(P)+8)-[(2 Q+6) \Theta(\infty, f) \\
\quad+(2+3 \underline{d}(P)) \delta(0, f)]\} T(r, f)+S(R, f) .
\end{aligned}
$$

Therefore, we have

$$
\begin{gathered}
\{(2 Q+6) \Theta(\infty, f)+(2+3 \underline{d}(P)) \delta(0, f) \\
-(2 Q+2 \bar{d}(P)+8)\} T(r, f) \leq S(r, f),
\end{gathered}
$$

which is a contradiction to our hypothesis (9).

Thus $H \equiv 0$. By integration, we get from (17) that

$$
\frac{1}{G-1}=\frac{A}{F-1}+B
$$

where $(A \neq 0)$ and $B$ are constants. Thus

$$
\begin{aligned}
& G=\frac{(B+1) F+(A-B-1)}{B F+(A-B)}, \\
& F=\frac{(B-A) G+(A-B-1)}{B G-(B+1)} .
\end{aligned}
$$

We discuss the following three cases.

Case 1. Suppose that $B \neq 0,-1$. From (46), we have

$$
\bar{N}\left(r, \frac{1}{G-(B+1) / B}\right)=\bar{N}(r, F) .
$$

From this and second fundamental theorem, we have

$$
\begin{aligned}
T(r, f) \leq & T(r, G)+S(r, f) \\
\leq & \bar{N}(r, G)+\bar{N}\left(r, \frac{1}{G}\right) \\
& +\bar{N}\left(r, \frac{1}{G-(B+1) / B}\right)+S(r, f) \\
\leq & \bar{N}(r, G)+\bar{N}\left(r, \frac{1}{G}\right)+\bar{N}(r, F)+S(r, f) \\
\leq & 2 \bar{N}(r, f)+\bar{N}\left(r, \frac{1}{f}\right)+S(r, f) \\
\leq & (2 Q+6) \bar{N}(r, f)+\left(2+3 \underline{d}(P) N\left(r, \frac{1}{f}\right)\right. \\
& +S(r, f) \\
\leq & \{(2 Q+3 \underline{d}(P)+8)-[(2 Q+6) \Theta(\infty, f) \\
& +(2+3 \underline{d}(P)) \delta(0, f)]\} T(r, f)+S(R, f) .
\end{aligned}
$$

Therefore, we have

$$
\begin{array}{r}
\{(2 Q+6) \Theta(\infty, f)+(2+3 \underline{d}(P)) \delta(0, f) \\
-(2 Q+3 \underline{d}(P)+7)\} T(r, f) \leq S(r, f),
\end{array}
$$

which is a contradiction to our hypothesis (9).

Case 2. Suppose that $B=0$, From (46), we get

$$
G=\frac{F+(A-1)}{A}, \quad F=A G-(A-1),
$$

we claim $A=1$.

If $A \neq 1$ from (50), we obtain

$$
N\left(r, \frac{1}{G-(A-1) / A}\right)=N\left(r, \frac{1}{F}\right) .
$$


From this, second fundamental theorem, and (20), we have

$$
\begin{aligned}
& T(r, f) \leq T(r, G)+S(r, f) \\
& \leq \bar{N}(r, G)+\bar{N}\left(r, \frac{1}{G}\right) \\
& +\bar{N}\left(r, \frac{1}{G-(A-1) / A}\right)+S(r, f) \\
& \leq \bar{N}(r, G)+\bar{N}\left(r, \frac{1}{G}\right)+N\left(r, \frac{1}{F}\right)+S(r, f) \\
& \leq \bar{N}(r, f)+N\left(r, \frac{1}{f}\right)+Q \bar{N}(r, f) \\
& +(\bar{d}(P)-\underline{d}(P)) m\left(r, \frac{1}{f}\right) \\
& +N\left(r, \frac{1}{f^{\bar{d}(P)}}\right)+S(r, f) \\
& \leq(Q+1) \bar{N}(r, f)+(1+\underline{d}(P)) N\left(r, \frac{1}{f}\right) \\
& +(\bar{d}(P)-\underline{d}(P)) T\left(r, \frac{1}{f}\right)+S(r, f) \\
& (1-\bar{d}(P)+\underline{d}(P)) T(r, f) \\
& \leq(2 Q+6) \bar{N}(r, f)+(2+3 \underline{d}(P)) N\left(r, \frac{1}{f}\right) \\
& +S(r, f) \\
& \leq\{(2 Q+3 \underline{d}(P)+8)-[(2 Q+6) \Theta(\infty, f) \\
& +(2+3 \underline{d}(P)) \delta(0, f)]\} T(r, f)+S(R, f) \text {. }
\end{aligned}
$$

Hence, we have

$$
\begin{aligned}
& \{(2 Q+6) \Theta(\infty, f)+(2+3 \underline{d}(P)) \delta(0, f) \\
& -(2 Q+2 \underline{d}(P)+\bar{d}(P)+7)\} T(r, f) \leq S(r, f),
\end{aligned}
$$

which is a contradiction to our hypothesis (9)

Thus, $A=1$.

From (50) we have $F \equiv G$.

Therefore, we have $f \equiv P[f]$.

Case 3.Suppose that $B=-1$, from (46) we have

$$
G=\frac{A}{-F+A+1}, \quad F=\frac{(1+A) G-A}{G} .
$$

If $A \neq-1$, we obtain from (54) that

$$
N\left(r, \frac{1}{G-A /(A+1)}\right)=N\left(r, \frac{1}{F}\right) .
$$

By the same argument as in Case 2, we obtain a contradiction. Hence, $A=-1$.
From (54), we get

$$
F G \equiv 1
$$

that is,

$$
f \cdot P[f] \equiv a^{2}
$$

From (57), we have

$$
N(r, f)+N\left(r, \frac{1}{f}\right)=S(r, f) .
$$

Using (54), (57), Lemma 12, and first fundamental theorem, we get

$$
\begin{aligned}
&(\bar{d}(P)+1) T(r, f) \\
&=T\left(r, \frac{1}{f^{\bar{d}(P)+1}}\right) \\
&=T\left(r, \frac{1}{f^{\bar{d}(P)} f}\right) \\
&=T\left(r, \frac{P[f]}{f^{\bar{d}(P)} a^{2}}\right)+S(r, f) \\
&=m\left(r, \frac{P[f]}{f^{\bar{d}(P)}}\right)+N\left(r, \frac{P[f]}{f \bar{d}(P)}\right)+S(r, f) \\
& \leq(\bar{d}(P)-\underline{d}(P)) m\left(r, \frac{1}{f}\right)+(\bar{d}(P)-\underline{d}(P)) N\left(r, \frac{1}{f}\right) \\
& \leq(\bar{d}(P)-\underline{d}(P)) T\left(r, \frac{1}{f}\right)+S(r, f) . \\
& \leq(\bar{d}(P)-\underline{d}(P)) m\left(r, \frac{1}{f}\right)+S(r, f) \\
&\left.+Q \bar{N}(r, f)+\bar{N}\left(r, \frac{1}{f}\right)\right]+S(r, f) \\
&=(r)
\end{aligned}
$$

From this, we have

$$
(\underline{d}(P))+1) T(r, f) \leq S(r, f),
$$

which is a contradiction. This completes the proof of Theorem 3.

Proof of Theorem 5. Let $F$ and $G$ be given by (26). From the assumption of Theorem 5, we know that $F$ and $G$ share $1 \mathrm{CM}$ :

$$
\overline{N_{L}}\left(r, \frac{1}{F-1}\right)=\overline{N_{L}}\left(r, \frac{1}{G-1}\right)=0 .
$$

Proceeding as in Theorem 3, we obtain (41). 
Using (61) in (41), we get

$$
\begin{aligned}
\bar{d}(P) & T(r, f) \\
\leq & 3 \bar{N}(r, G)+(\bar{d}(P)-\underline{d}(P)) m\left(r, \frac{1}{f}\right) \\
& +N\left(r, \frac{1}{f \bar{d}(P)}\right)+N\left(r, \frac{1}{f}\right)+S(r, f) \\
\leq & 3 \bar{N}(r, f)+(\bar{d}(P)-\underline{d}(P))\left(T(r, f)-N\left(r, \frac{1}{f}\right)\right) \\
& +(\bar{d}(P)+1) N\left(r, \frac{1}{f}\right)+S(r, f), \\
\underline{d}(P) T(r, f) & \\
\leq & \bar{N}(r, f)+(\underline{d}(P)+1) N\left(r, \frac{1}{f}\right)+S(r, f) \\
\leq & \{(\underline{d}(P)+4) \\
& -[3 \Theta(\infty, f)+(\underline{d}(P)+1) \delta(0, f)]\} T(r, f)+S(r, f) .
\end{aligned}
$$

We have

$$
\{3 \Theta(\infty, f)+(\underline{d}(P+1)) \delta(0, f)-4\} T(r, f) \leq S(r, f),
$$

which contradicts (10).

Thus, $H \equiv 0$. Proceeding as in Theorem 3, we prove Theorem 5.

Proof of Theorem 7. $f$ is a nonconstant entire function. Taking $N(r, f)=0$ in proof of Theorem 3, we obtain Theorem 7 .

Proof of Theorem 9. $f$ is a nonconstant entire function. Taking $N(r, f)=0$ in proof of Theorem 5, we obtain Theorem 9 .

\section{Acknowledgments}

The authors thank the referee for his/her valuable suggestions. This research work is supported by the Department of Science and Technology Government of India, Ministry of Science and Technology, Technology Bhavan, New Delhi, India, under the sanction Letter no. (SR/S4/MS: 520/08).

\section{References}

[1] H. X. Yi and C. C. Yang, Uniqueness Theory of Meromorphic Functions, Science Press, Beijing, China, 1995.

[2] W.-C. Lin and H.-X. Yi, "Uniqueness theorems for meromorphic function," Indian Journal of Pure and Applied Mathematics, vol. 35, no. 2, pp. 121-132, 2004.

[3] R. Brück, "On entire functions which share one value CM with their first derivative," Results in Mathematics, vol. 30, no. 1-2, pp. 21-24, 1996.
[4] K.-W. Yu, "On entire and meromorphic functions that share small functions with their derivatives," Journal of Inequalities in Pure and Applied Mathematics, vol. 4, no. 1, article 21, 2003.

[5] L. Liu and Y. Gu, "Uniqueness of meromorphic functions that share one small function with their derivatives," Kodai Mathematical Journal, vol. 27, no. 3, pp. 272-279, 2004.

[6] T. Zhang and W. Lü, "Notes on a meromorphic function sharing one small function with its derivative," Complex Variables and Elliptic Equations, vol. 53, no. 9, pp. 857-867, 2008.

[7] S. S. Bhoosnurmath and A. J. Patil, "On the growth and value distribution of meromorphic functions and their differential polynomials," The Journal of the Indian Mathematical Society, vol. 74, no. 3-4, pp. 167-184, 2007.

[8] H.X. Yi, "Uniqueness of meromorphic functions and a question of C. C. Yang," Complex Variables-Theory and Application, vol. 14, no. 1-4, pp. 169-176, 1990.

[9] H.-X. Yi, "Uniqueness theorems for meromorphic functions whose n-th derivatives share the same 1-points," Complex Variables-Theory and Application, vol. 34, no. 4, pp. 421-436, 1997.

[10] N. Li and L.-Z. Yang, "Meromorphic function that shares one small function with its differential polynomial," Kyungpook Mathematical Journal, vol. 50, no. 3, pp. 447-454, 2010. 


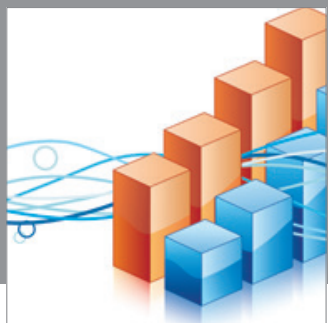

Advances in

Operations Research

mansans

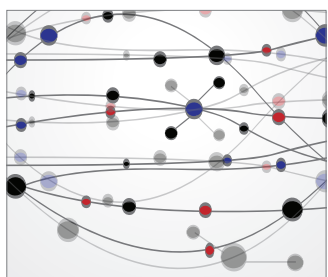

The Scientific World Journal
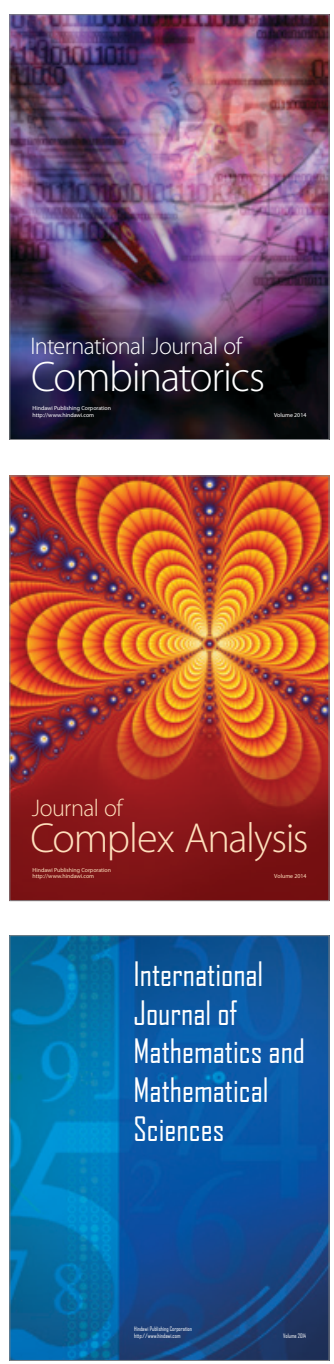
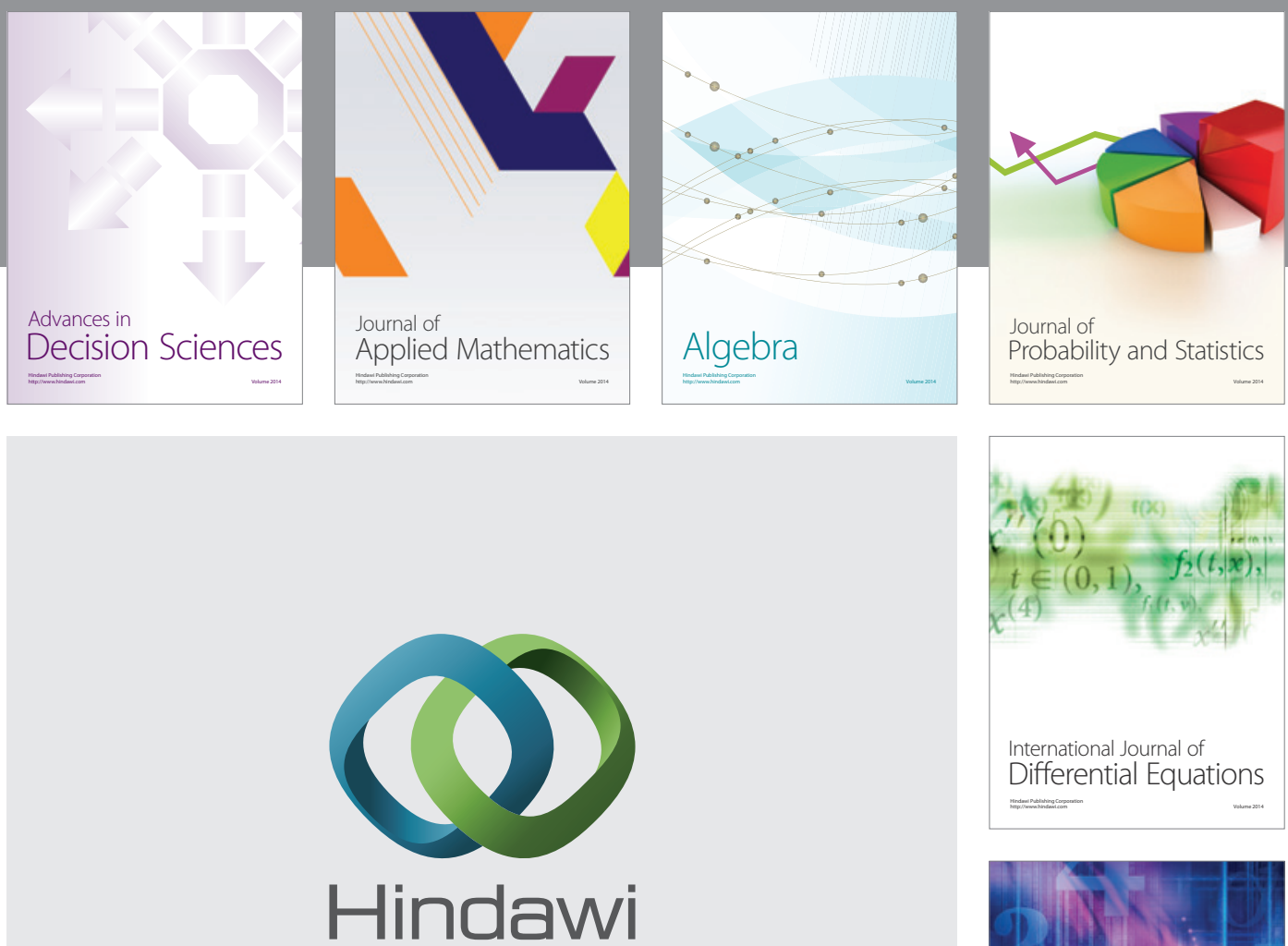

Submit your manuscripts at http://www.hindawi.com
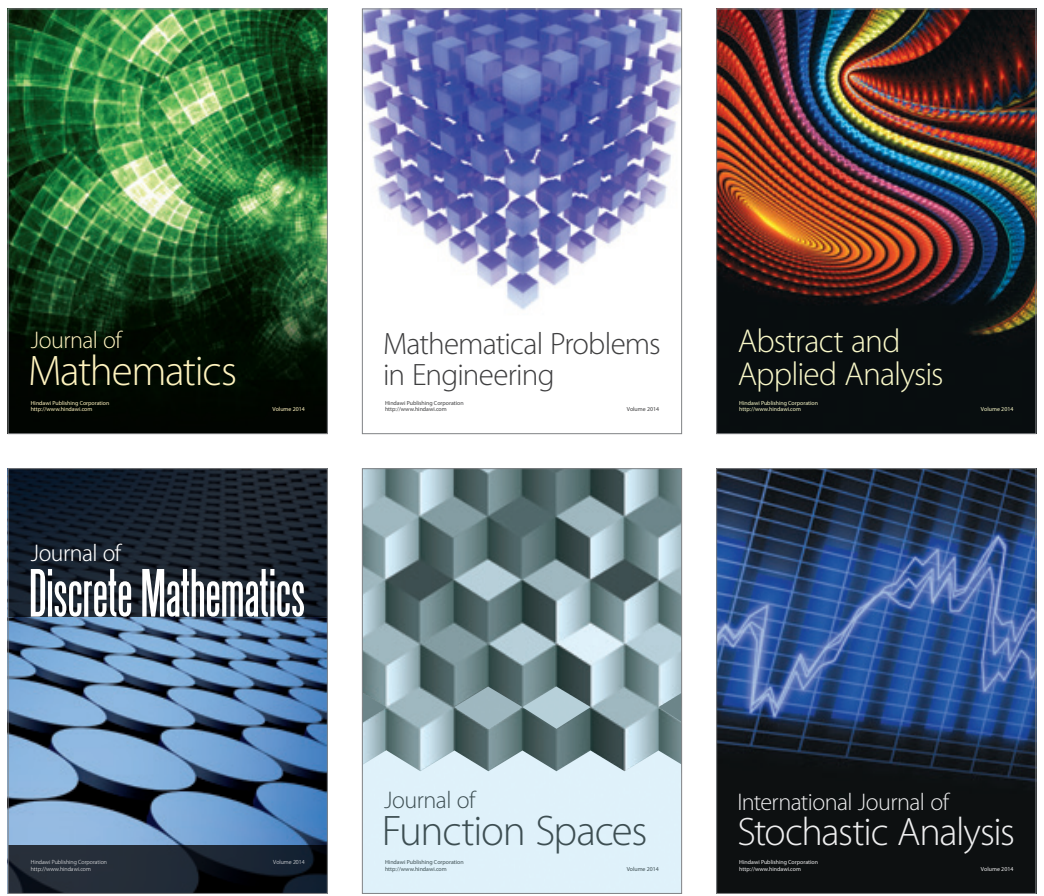

Journal of

Function Spaces

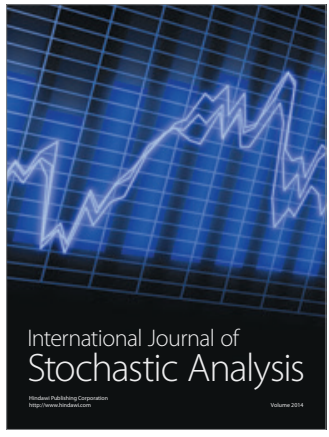

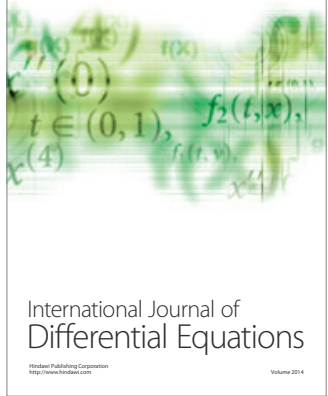
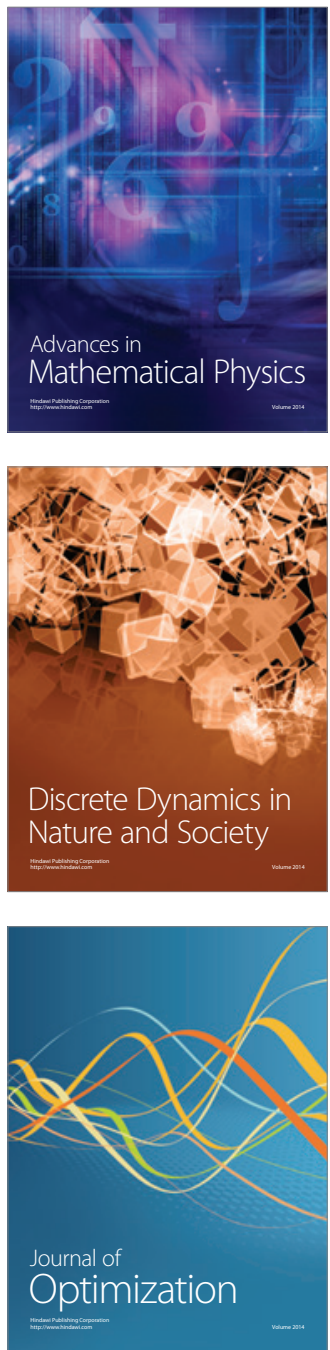